\title{
Políticas energéticas en la UE: seguridad de suministro, medio ambiente y competencia
}

\author{
Giulio Federico \\ Xavier Vives \\ Centro Sector Público-Sector Privado \\ IESE Business School (Universidad de Navarra)
}

\begin{abstract}
Resumen
Este artículo estudia el estado actual de la política energética de la UE examinando sus tres pilares principales: la seguridad de suministro, el medio ambiente y la competencia. Se revisan las últimas tendencias en los tres ámbitos y presenta una evaluación a corto y medio plazo de las perspectivas de evolución de la dependencia energética exterior y de los cambios que se requieren para cumplir con los objetivos medioambientales. El artículo sostiene que las políticas para reducir la dependencia energética pueden llegar a ser complementarias a las necesarias para reducir las emisiones de dióxido de carbono en el futuro, y que será necesaria una transformación importante del sector eléctrico en particular, para cumplir con los actuales objetivos de cambio climático. Una regulación y competencia efectiva seguirán siendo, en el mercado energético interno de la UE, instrumentos fundamentales de política para contener el coste de la energía en la transición hacia un sector de bajas emisiones de dióxido de carbono.
\end{abstract}

Palabras Clave: dependencia energética, cambio climático, regulación, competencia, defensa de la competencia.

Clasificación JEL: L40, L51, Q40, Q48, Q54.

\begin{abstract}
This article surveys the current state of EU energy policy by examining its three main pillars: security of supply, environment, and competition. We review the latest trends in all three areas and provide an assessment of the short to medium term prospects for the evolution of external energy dependence and of the future changes required to comply with environmental objectives. We argue that policies to reduce energy dependence may become complementary to those required to reduce carbon emissions in the future, and that a significant transformation of the electricity sector in particular will be required to meet the current climate change targets. Effective regulation and competition in the internal EU energy market will remain a key policy tool to contain the cost of energy in the transition to a low-carbon sector.
\end{abstract}

Keywords: Energy dependence, climate change, regulation, competition, antitrust.

JEL Classification: L40, L51, Q40, Q48, Q54.

${ }^{1}$ Este artículo se basa en parte en el capítulo sobre el contexto energético europeo del informe "Competencia y Regulación en los Mercados Españoles del Gas y la Electricidad", del Centro Sector Público-Sector Privado (SP-SP) de IESE Business School (publicado en el 2008). Agradecemos los comentarios de Ángel López y la ayuda de investigación de Fernanda Gutiérrez en la preparación del artículo, así como el apoyo financiero del Ministerio de Ciencia e Innovación a través del proyecto ECO2008-05155 en el Centro SP-SP. Vives agradece también la colaboración de la Cátedra Abertis de Regulación, Competencia y Políticas Públicas. 
El sector energético europeo está experimentando una transformación importante y se enfrenta a una serie de desafíos difíciles. Estos incluyen una elevada y creciente dependencia de las fuentes de energía externas, la necesidad de reducir drásticamente las emisiones de gases de efecto invernadero para cumplir con los objetivos medioambientales, y las dificultades relacionadas con la promoción de un mercado de la energía efectivamente integrado y competitivo. Algunas de las políticas asociadas con los distintos objetivos a veces están en conflicto entre sí, mientras que en otros casos se refuerzan mutuamente.

Este breve artículo analiza las principales tendencias en los sectores de gas y electricidad en Europa, centrándose principalmente en los tres pilares de la política energética en la UE: seguridad de suministro; medio ambiente y cambio climático; y promoción de un mercado interno competitivo e integrado. Las primeras tres secciones del artículo analizan las últimas tendencias en relación con cada uno de estos tres pilares. La cuarta sección discute los principales retos futuros para la política europea en los sectores del gas y la electricidad.

\section{La dependencia de energía en Europa y la seguridad de suministro}

\subsection{Dependencia de energía importada}

La disponibilidad de fuentes energéticas seguras y eficientes es uno de los retos principales que enfrenta el mercado europeo de la energía. El sector energético europeo se ha caracterizado por una dependencia creciente de fuentes de energía importada, que se debe, en gran medida, a las importaciones de petróleo y gas. Esta dependencia aumentó del 44\% en 1990 al 53\% en 2007 para la UE-27 (véase Figura 1). Los valores correspondientes para la UE-15 y (especialmente) para España son mayores y también siguen una tendencia creciente (en el caso de España la dependencia aumentó de más del 60\% en 1990 a casi el 80\% en 2007).

Los sectores del gas y la electricidad que se analizan en este artículo no dependen directamente del petróleo de manera significativa. Para estos mercados es más importante la dependencia del gas que la dependencia del petróleo. Sin embargo, dado el fuerte vínculo que todavía existe entre los precios de las importaciones de gas y petróleo, las tendencias en el mercado global del petróleo tienen consecuencias directas en el precio del gas importado.

La mayor dependencia del gas está motivada por el rápido aumento en el consumo en los últimos años, que se debe, en parte, al aumento en la proporción de generación eléctrica que utiliza gas. En la UE la cuota de generación eléctrica mediante gas aumentó del 15\% al 23\% en diez años (entre 1998 y 2007, véase Figura 2). En España este aumento es incluso más pronunciado.

El aumento de la demanda de gas en Europa, junto con el estancamiento de su producción (principalmente en Reino Unido y Holanda), ha centrado la atención en las fuentes de importación de gas. Como se muestra en la Figura 3, Rusia es el principal 
FIGURA 1

EVOLUCIÓN DE LA DEPENDENCIA DE ENERGÍA IMPORTADA, 1990-2007

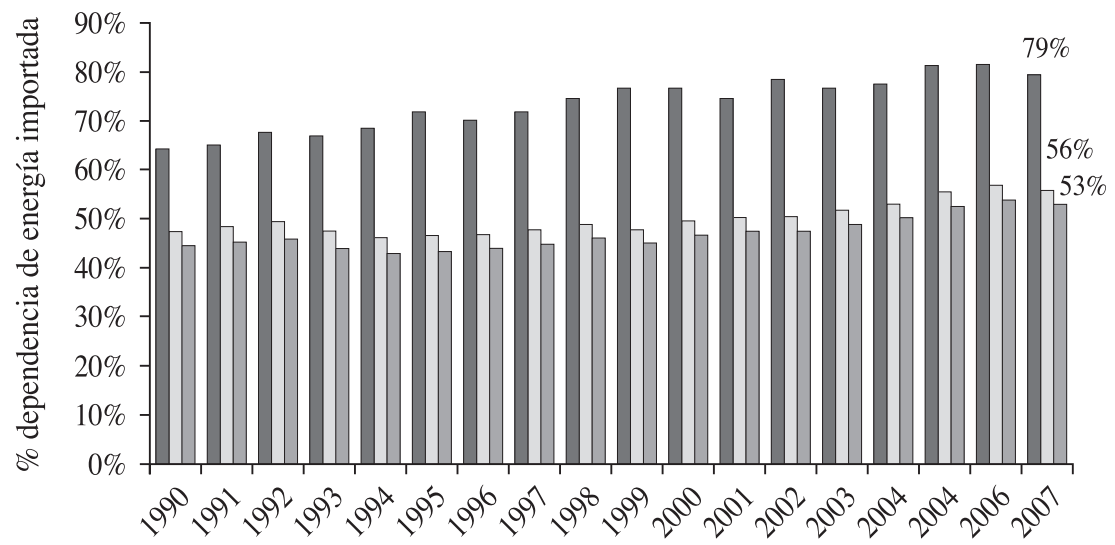

España $\square$ UE-15 $\square$ UE-27

Fuente: Eurostat.

FIGURA 2

MIX DE GENERACIÓN EN UE-27 Y ESPÃ̃A, 1998-2007

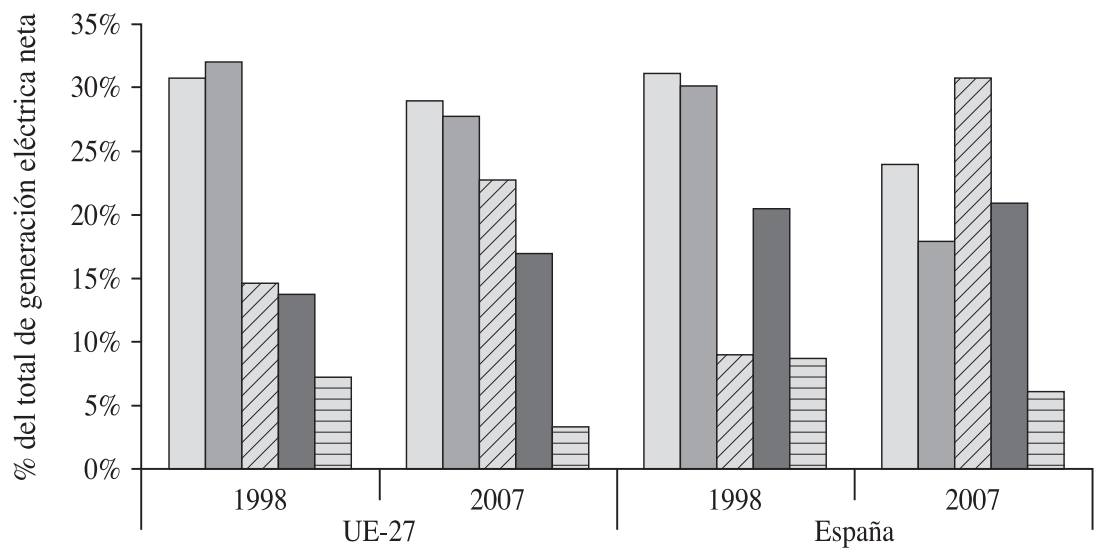

$\square$ Carbón $\square$ Nuclear $\square \not \triangle$ Gas $\square$ Renovables $\quad \square$ Petróleo 
proveedor de gas a países de la UE-27 (representando un 31\% de la suma de las importaciones de todos los países miembros, y $41 \%$ de las importaciones provenientes de países no-miembros de la UE), seguida de Noruega (20\% y $27 \%$ respectivamente) y de Argelia (13\% y $17 \%$ respectivamente). La dependencia del gas ruso ha bajado desde 1990 en términos relativos, en parte gracias al aumento de las importaciones provenientes de otras fuentes (principalmente GNL), pero aún así sigue siendo elevada.

\section{FIGURA 3}

\section{DEPENDENCIA DEL GAS IMPORTADO EN LA UE-27, INCLUYENDO IMPORTACIONES INTRA-UE}

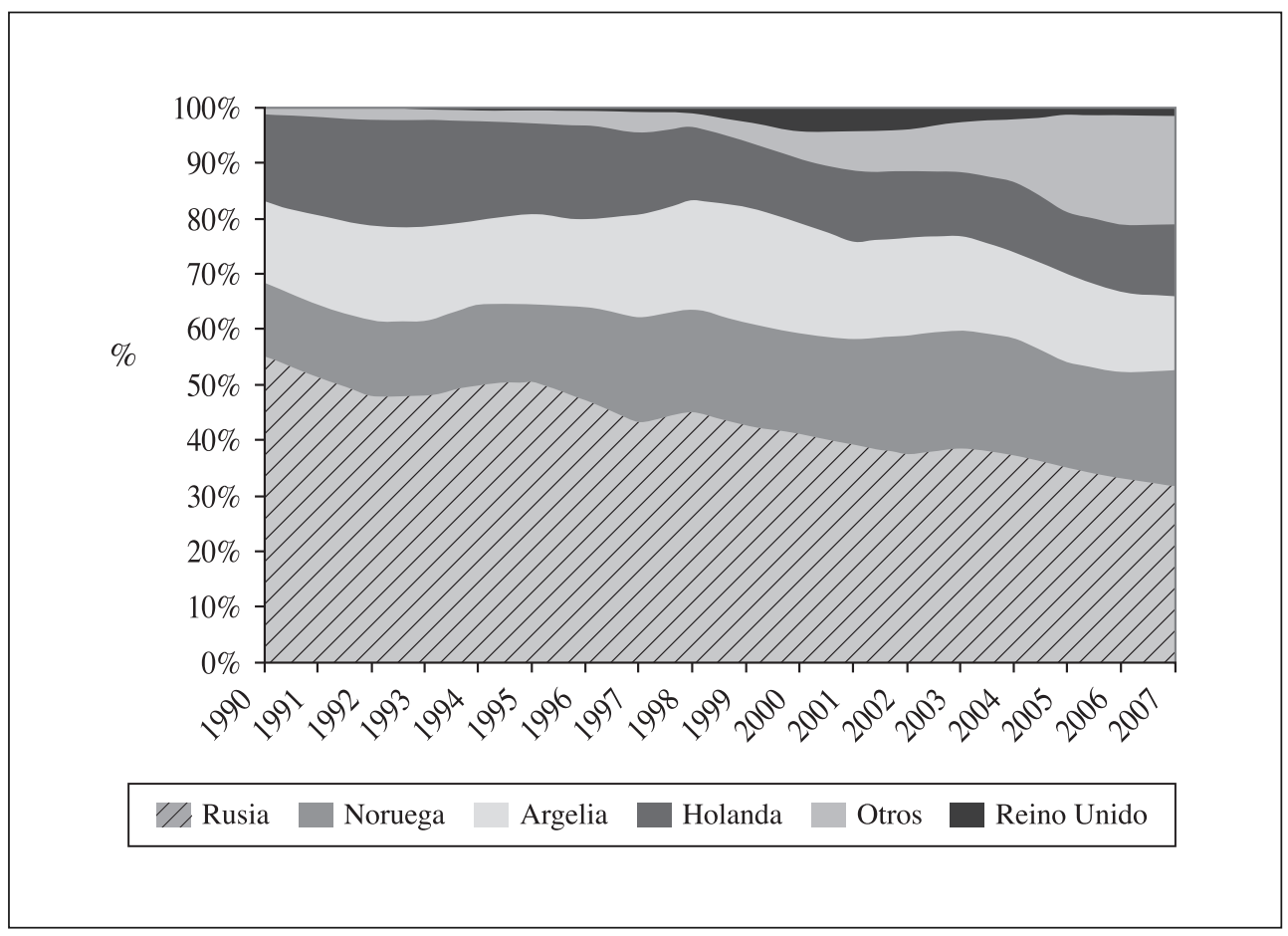

Fuente: Eurostat.

En los cinco principales países de la UE hay variaciones importantes tanto en el nivel de la dependencia del gas importado como en el mix y la concentración de las importaciones. Alemania, Italia, Francia y España dependen casi exclusivamente del gas importado para su consumo (en contraste con el Reino Unido), y la concentración por país importador es particularmente elevada en Alemania debido a su dependencia de gas ruso. En España destacan también la ausencia de gas ruso en las importaciones y el peso elevado de sus importaciones de GNL (más del 70\% de las importaciones en el 2008, frente a un $18 \%$ para la UE-15). 


\subsection{Tendencias futuras}

La dependencia europea del gas importado está destinada a aumentar en el futuro próximo. El último World Energy Outlook (WEO) de la Agencia Internacional de Energía - publicado en noviembre de 2009- pronostica para la UE un aumento de la dependencia de gas del 60\% en 2007 y superior al $80 \%$ en 2030. En los dos escenarios elaborados en el WEO-2009, las importaciones de gas suben entre 125 y $200 \mathrm{bcm}^{2}$ hasta el 2030 (una subida entre el $37 \%$ y el 65\% respecto al 2007) y la producción doméstica de gas baja entre el $52 \%$ y el $62 \%$ en el mismo periodo (equivalente a unos 110-130 bcm).

Los niveles actuales de reservas de gas de los principales países proveedores de Europa (Rusia, Noruega, Argelia, Holanda y Reino Unido) revelan el papel clave que jugará Rusia como fuente creciente de importaciones de gas a través de gasoductos en el futuro. Frente a las reservas de Rusia, las reservas agregadas de los otros países importantes proveedores de gas de la UE son pequeñas. Las reservas rusas actualmente ascienden a $43 \mathrm{tcm}^{3}$, mientras que las reservas de gas de Argelia, Noruega, Holanda y Reino Unido en conjunto no alcanzan los 10 tcm. Sin embargo, las reservas de algunos países exportadores de GNL pueden equipararse a las de Rusia, poniendo de manifiesto el papel potencialmente crucial que podrían jugar las importaciones de GNL como alternativa competitiva al gas proveniente de Rusia ${ }^{4}$.

Se prevé la construcción de varios gasoductos nuevos para cubrir la mayor dependencia de gas importado en Europa a medio plazo. La Tabla 1 en al Anexo resume los proyectos de gasoductos que el último Plan Prioritario Europeo de Interconexión considera más probables (y también incluye el proyecto South Stream, que no está incluido en el Plan). El aumento de la capacidad de importación podría llegar aproximadamente a $100 \mathrm{bcm}$ (con un potencial aumento superior a los $180 \mathrm{bcm}$, si se completa el desarrollo de todos los proyectos). Una parte considerable de la nueva capacidad adicional (más del 60\%) puede ser proporcionada por Rusia, debido al desarrollo potencial de dos grandes gasoductos (North Stream y South Stream). Otros gasoductos potenciales (principalmente Nabucco) suministrarán gas proveniente de otras fuentes localizadas en Asia Central y en el Caspio, a pesar de que la composición exacta de este gas adicional y su potencial fiabilidad son todavía inciertas ${ }^{5}$. La

${ }^{2}$ Billion cubic meters ( 1 bcm es equivalente a mil millones de metros cúbicos. Es una medida estándar en el mercado del gas).

${ }^{3}$ Trillion cubic meters ( $1 \mathrm{tcm}$ es equivalente a $1.000 \mathrm{bcm}$ ).

${ }^{4}$ De acuerdo con los datos publicados por $B P$, los países que en la actualidad sólo exportan GNL a Europa tenían reservas de 34 tcm a finales de 2008, equivalente al 79\% de las reservas de Rusia. La mayor parte de estas reservas provienen de Qatar $(25 \mathrm{tcm})$. El otro posible exportador a Europa con grandes reservas de gas es Irán, con 30 tcm.

${ }^{5}$ Sin embargo, proyecciones del regulador Británico Ofgem indican que la dependencia del gas ruso aumentará en el 2020 (hasta el 55\% de las importaciones, incluso en escenarios con políticas medioambientales eficaces) -véase Ofgem, Project Discovery. Energy Market Scenarios, octubre de 2009. Estas proyecciones también indican que el peso del GNL en las importaciones de la UE aumentará hasta el $25-30 \%$ en 2020. 
Comisión Europea también pronosticaba un aumento considerable en la capacidad de importación de GNL, desde aproximadamente 80 bcm a comienzos de 2007 a 135-140 bcm para 2010-2012.

En conjunto, el posible aumento de la capacidad de gasoductos y GNL en el medio plazo excede el aumento de las importaciones previsto en el WEO 2009 para el año 2030, especialmente en el escenario con políticas de ahorro energético más eficaces. La competitividad y la seguridad en el mercado europeo del gas aumentarían si se consiguiera un mix equilibrado de los proyectos de importación de gas, con una combinación de importaciones tanto de GNL como de gas a través de gasoductos de nuevas fuentes (por ejemplo, distintas de Rusia y Argelia).

\section{Políticas medioambientales, cambio climático e implicaciones para el mercado energético europeo}

Las políticas medioambientales en el contexto del cambio climático representan el segundo pilar de la política energética en Europa. La necesidad de reducir la emisión de gases de efecto invernadero (GEI) para evitar un calentamiento global excesivo ha sido un factor determinante de la política energética en Europa en los últimos tiempos, y llegará a ser aún más crítica en el medio y largo plazo. El sector energético europeo tendrá que emprender una profunda transformación si quiere cumplir con los objetivos propuestos.

\subsection{La política medioambiental hasta la fecha}

El elemento principal de la política medioambiental en Europa, hasta la fecha, ha sido el Protocolo de Kioto (y las políticas asociadas al mismo). En el marco del Protocolo la UE (definida como la UE-15) se ha comprometido a reducir durante el periodo 2008-2012 sus emisiones de gases de efecto invernadero en un $8 \%$ con respecto a los niveles de 1990. Las emisiones durante el periodo 2004-2008 de la UE-15 fueron un 4\% inferiores a los niveles de 1990, y las últimas proyecciones publicadas por la European Environment Agency (EEA) indican que el objetivo marcado por el Protocolo de Kioto será alcanzado gracias a las reducciones significativas de las emisiones en la antigua Alemania del Este (después de la Reunificación a comienzos de los noventa), y a las de Francia y Reino Unido. Estas reducciones son, sin embargo, parcialmente compensadas por el aumento significativo de las emisiones en España respecto a su objetivo de Kioto.

Una de las principales políticas relacionadas con el Protocolo de Kioto ha sido la introducción del Emission Trading System (ETS) en Europa, a partir de 2005. El ETS es un mecanismo de "cap-and-trade" que establece un precio para el dióxido de carbono $\left(\mathrm{CO}_{2}\right)$, y que permite a los mayores emisores de $\mathrm{CO}_{2}$ comerciar derechos de emisión con el fin de fomentar una reducción eficiente de las mismas. En 
la actualidad abarca instalaciones que en conjunto representan el $40 \%$ de las emisiones de gases de efecto invernadero de la UE. El ETS ha conseguido establecer un precio para el $\mathrm{CO}_{2}$ de forma transparente, $\mathrm{y}$ ha permitido su comercio, a pesar de las legítimas preocupaciones que se han expresado acerca de la excesiva generosidad en la emisión de derechos en la Fase I (2005-2007); y a sus consecuencias en la distribución de las ganancias entre productores y consumidores, debido a la asignación de derechos de emisión gratuitos durante las fases iniciales del mecanismo (la subasta completa de derechos de emisión se iniciará sólo en la Fase III, que empezará en 2013).

Otra política medioambiental relacionada con la necesidad de cumplir con los objetivos del Protocolo de Kioto ha sido el fomento (y subvención) de las fuentes renovables en el sector de generación eléctrica. Esto ha dado lugar a un aumento moderado de la generación de energía renovable en el pasado reciente, todavía muy por debajo de los objetivos fijados. Como se muestra en la Figura 4, en 2007 se-

FIGURA 4

CUOTA DE ELECTRICIDAD RENOVABLE EN LA GENERACIÓN TOTAL, 1990-2007

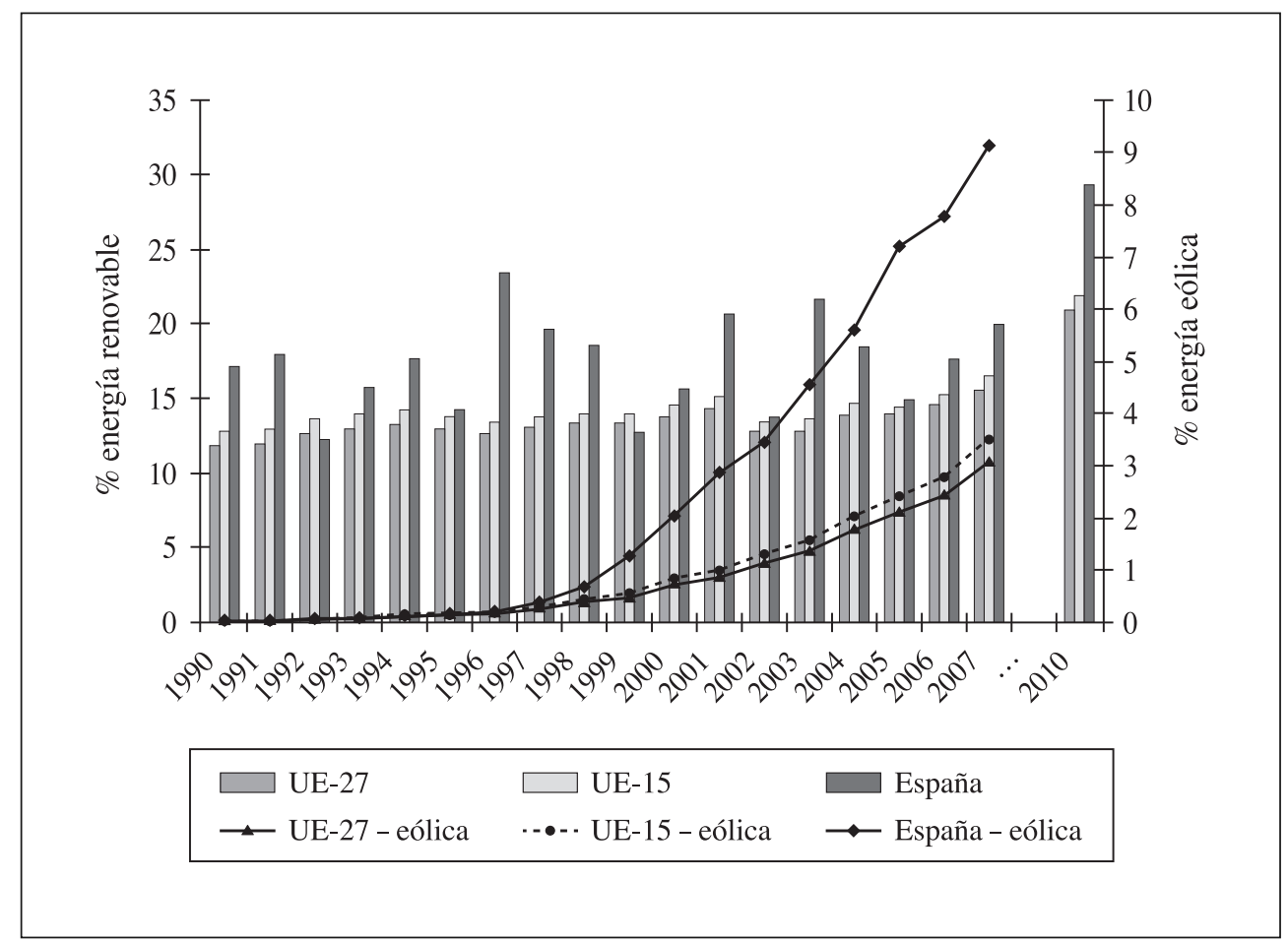

Fuente: Eurostat.

Nota: La definición de energía renovable incluye energía hidráulica, pero excluye bombeo. 
gún datos de Eurostat en Europa las fuentes renovables representaron poco más del $15 \%$ de la demanda total de electricidad, frente al objetivo europeo del $21 \%$ fijado para 2010. España alcanzó un nivel superior de energía renovable en el 2007 (20\%), en parte gracias a los progresos significativos alcanzados en la generación eólica (que representó el 9\% de la demanda en España en 2007, muy por encima del nivel europeo del 3-4\%). En 2009 debido al incremento continuo de generación renovable en régimen especial y a la caída de la demanda, estimamos que España alcanzó un $25 \%$ de energía renovable, relativamente cerca de su meta del 29\% fijada para el 2010.

El crecimiento del uso del gas y de la generación de energía renovable en el sector eléctrico ha reducido la intensidad de $\mathrm{CO}_{2}$ en la producción eléctrica (desde aproximadamente $600 \mathrm{t} \mathrm{CO}_{2} / \mathrm{GWh}$ en 1990 a menos de $450 \mathrm{t} \mathrm{CO}_{2} / \mathrm{GWh}$ en 2006). Este progreso hacia una menor generación de energía basada en el $\mathrm{CO}_{2}$ es, sin embargo, insuficiente para cumplir con las ambiciosas metas de la política del cambio climático de la UE en el medio plazo, tal como veremos en la siguiente subsección.

\subsection{Políticas y tendencias futuras}

En 2008 se aprobó un nuevo Paquete Europeo de Medidas Climáticas y Energéticas (también conocido como el Plan 20-20-20) que establece nuevos objetivos para el 2020. Estos incluyen:

i) una reducción del $20 \%$ de las emisiones de GEI respecto a 1990 (muy por encima del objetivo de Kioto del 8\%);

ii) alcanzar una cuota del $20 \%$ de fuentes renovables en el consumo total de energía $^{6}$ (de nuevo, muy por encima del objetivo actual del $12 \%$ en 2010 , y de los actuales niveles inferiores al 9\%), y

iii) una mejora en la eficiencia energética de un $20 \%$.

El paquete de medidas incluye un compromiso para aumentar la reducción de emisiones del $20 \%$ al $30 \%$ en el contexto de un acuerdo internacional cooperativo sobre el clima en el que otros países desarrollados y en desarrollo contribuyan a limitar sus emisiones.

Dos nuevas Directivas fueron aprobadas en 2009 para implementar este plan: una Directiva sobre Energías Renovables (2009/28/CE), que fija objetivos nacionales de energía renovables: y una Directiva (2009/29/CE) que reforma el ETS para el período post Kioto incluyendo, en particular, una reducción del $21 \%$ de los derechos de emisión dentro del ETS en 2020, en relación con 2005, y una disposición para la su-

${ }^{6}$ Este objetivo implica para la UE una cuota de energías renovables en el consumo de electricidad de alrededor de 33\% (y todavía más en algunos países miembros como España). 
basta completa de derechos de emisión para el sector eléctrico a partir de 2013 de aplicación en principio a la mayoría de Estados miembros.

Los objetivos establecidos en el nuevo paquete de medidas son ambiciosos, y requerirán cambios importantes en la manera en que los mercados de energía europeos operan. El WEO-2009 muestra que incluso cumplir con estos objetivos posiblemente no será suficiente para evitar un aumento de la temperatura global superior a los 2 grados Celsius ${ }^{7}$. El WEO-2009 desarrolla un escenario donde el stock global de emisiones de GEI en la atmósfera finalmente se estabiliza a 450 partes por millón de $\mathrm{CO}_{2}$ equivalente (el escenario "450"), para cumplir los objetivos medioambientales. Bajo este escenario, las emisiones de $\mathrm{CO}_{2}$ del sector energético europeo en su conjunto y del sector eléctrico se deben reducir respectivamente en un $23 \%$ y un tercio con relación a los niveles de 1990 (por encima de los objetivos de reducción aprobados por la UE).

Para lograr estos objetivos, el sector eléctrico tendrá que reducir drásticamente su intensidad en las emisiones de $\mathrm{CO}_{2}$ de aquí a 2020, desde los niveles actuales cercanos a las $450 \mathrm{t} \mathrm{CO}_{2} / \mathrm{GWh}$ a $275 \mathrm{t} \mathrm{CO}_{2} / \mathrm{GWh}$. Como ilustra la Figura 5, esto a su vez requerirá (en relación a los niveles de 2007) una reducción significativa del peso de las centrales de carbón tradicionales, una cuota estable de gas, la preservación de la cuota actual de la generación de energía nuclear (cercana al 30\%) , y un aumento importante de otras tecnologías que no emiten $\mathrm{CO}_{2}$ (hidráulica, eólica y otras energías renovables) del 15\% actual al 32\%. La Figura 5 también muestra el mix de generación eléctrica en España en el 2008. Como puede observarse, en comparación con el resto de la UE, España ha reemplazado más carbón hacia el gas, y su menor dependencia de la energía nuclear se ve compensada con una mayor cuota de generación eólica. España necesita aumentar la participación total de energías que no emitan $\mathrm{CO}_{2}$, que incluye la nuclear, para el año 2020. La actual dependencia de las centrales de gas tanto en Europa como en España no será suficiente para contener las emisiones en el futuro, dada la necesidad de alcanzar una intensidad media de emisiones de $\mathrm{CO}_{2}$ para el mercado que está por debajo de los niveles asociados con las plantas de gas (que son de aproximadamente $365 \mathrm{t} \mathrm{CO}_{2} / \mathrm{GWh}$ ).

${ }^{7}$ El WEO-2009 considera necesario contener el aumento de la temperatura global en 2 grados Celsius por encima de los niveles pre-industriales para prevenir un calentamiento global que se considera excesivo. Este umbral de 2 grados está en consonancia con el acuerdo alcanzado en Copenhague en diciembre de 2009.

${ }^{8}$ Dado el aumento previsto de la demanda de electricidad durante el período 2007-2020, estas proyecciones implican un ligero aumento en la producción de las centrales nucleares (aproximadamente en un 5\%), lo que implica la necesidad de sustituir los reactores nucleares que se cierren y añadir nueva capacidad nuclear. 


\section{FIGURA 5}

\section{CAMBIO CLIMÁTICO E IMPLICACIONES PARA EL MIX DE GENERACIÓN ELÉCTRICA (UE-27 Y ESPAÑA)}

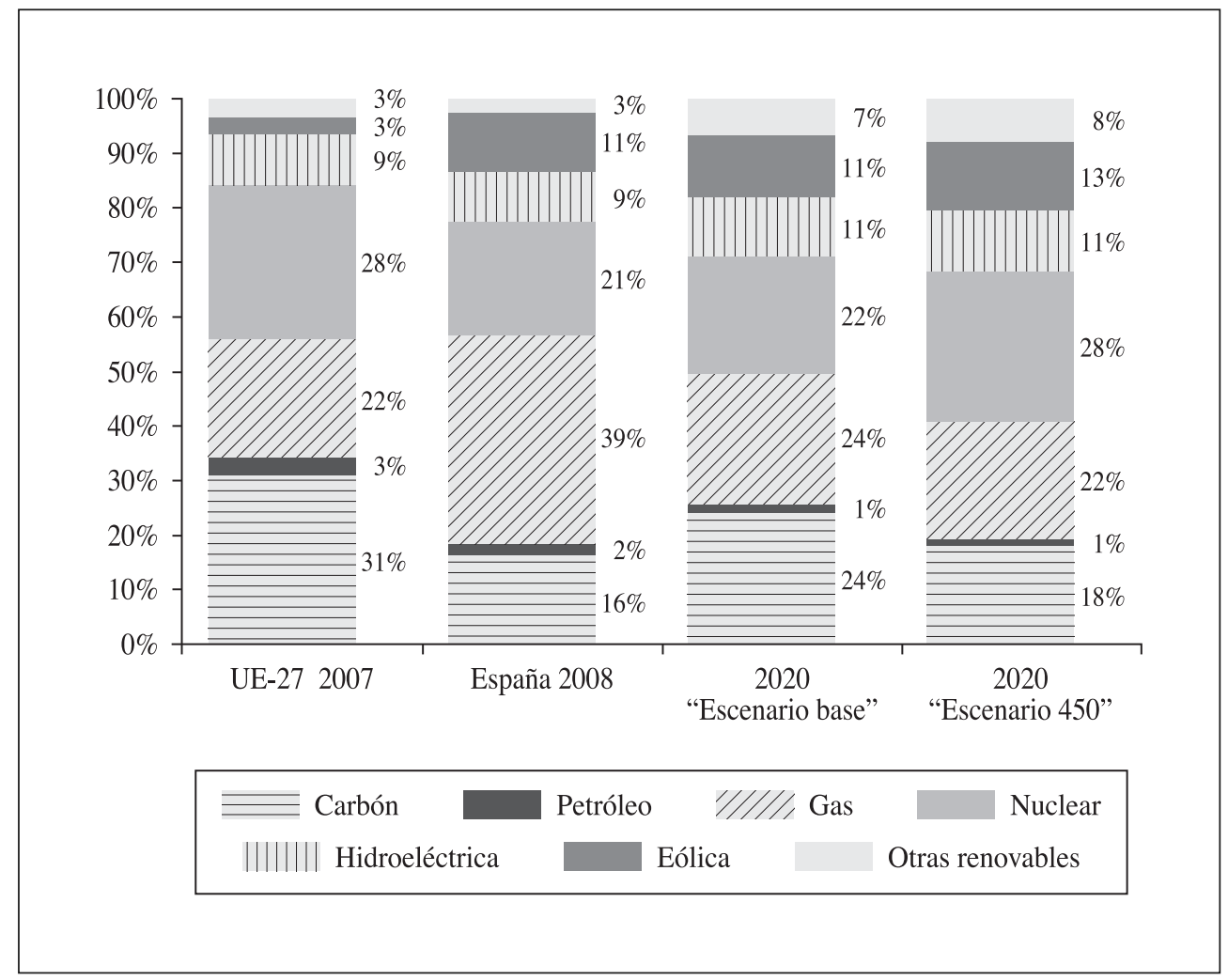

Fuente: World Energy Outlook, 2009; y Red Eléctrica de España, 2008.

\section{Políticas de regulación y de competencia en el sector energético europeo}

La aplicación de políticas eficaces de regulación y competencia sigue siendo una de las principales prioridades de la Comisión Europea en el sector energético, con el objetivo de lograr un mercado interno competitivo e integrado. En esta sección del artículo se examinan las últimas tendencias en este área.

La reforma regulatoria y la política de defensa de la competencia han sido áreas de intensa actividad desde la publicación a principios de 2007 de las conclusiones de la encuesta sobre el Sector Energético. Esta encuesta analizó tanto el sector de la electricidad como el del gas, e identificó una serie de problemas competitivos, como concentración excesiva, posible cierre de mercados mayoristas y minoristas, débil integración del mercado, y falta de transparencia. 


\subsection{Concentración e integración de mercados}

El indicador más usual para identificar posibles problemas de competencia es el grado de concentración en los segmentos de mercado liberalizados (generación y suministro minorista). Los datos de la Comisión Europea ${ }^{9}$ para el 2007 muestran que los niveles de concentración en la mayoría de los mercados europeos claves siguen siendo elevados tanto en generación como en suministro.

Parte de las cuestiones horizontales de competencia en el sector se derivan de mercados que suelen ser nacionales (especialmente en periodos de demanda punta) debido a la capacidad relativamente limitada de interconexión entre los Estados miembros. Este problema es particularmente grave en la electricidad, debido a las dificultades que presenta el transporte de la energía eléctrica a distancias considerables. Las proyecciones de 2009 publicadas por la $\mathrm{UCTE}^{10}$ indican que en países como el Reino Unido y España, la capacidad de importación de electricidad está muy por debajo del $10 \%$ de la demanda pico, mientras que en sistemas mejor interconectados, como Alemania, Italia y Francia se encuentra en el rango de 13-20\%. Sólo en sistemas más pequeños y muy integrados como el belga y el neerlandés, la capacidad de importación alcanza niveles que están por encima del 20-25\%.

\subsection{Separación de las redes de transporte y Directivas de 2009}

Otra preocupación clave señalada en la Encuesta del 2007 es el cierre de mercado a través de la discriminación ejercida por los incumbentes integrados verticalmente y mediante los contratos a largo plazo. La Comisión Europea considera que la falta de una separación efectiva de la red es uno de los factores más importantes detrás del cierre de mercado, a pesar de las disposiciones de las Directivas de gas y electricidad de 2003 (que sin embargo permiten a las entidades que tienen presencia en la producción y/o suministro, la propiedad y control de los activos de la red) ${ }^{11}$.

Una de las medidas regulatorias clave, propuesta por la Comisión Europea en el tercer paquete legislativo presentado en septiembre de 2007, consistía en la segregación de las redes en todos los Estados miembro como la opción regulatoria preferida. Las propuestas también ofrecen una opción alternativa en la forma de un "operador del sistema independiente" (Independent System Operator - ISO). Debido a la oposición de algunos Estados miembros (liderados por Francia y Alemania) a las propuestas legislativas de la Comisión Europea en favor de la separación de la propiedad de los activos de las redes energéticas, la versión final de las nuevas Directivas de

${ }^{9}$ Véase Comisión Europea, Report on Progress in Creating the Internal Gas and Electricity Market, marzo de 2009.

${ }^{10}$ Union for the Coordination of Transmission of Electricity.

${ }^{11}$ En la actualidad, una minoría de los Estados miembro de la UE-15 (5 de los 15 países) ha impuesto la separación de la propiedad de sus redes de gas, mientras que una pequeña mayoría ( 8 de los 15 países) ha implementado esta medida en el sector de la electricidad. 
Gas y de Electricidad (adoptadas en julio de 2009) incluyen una tercera opción de compromiso, basada en una regulación más estricta de los incumbentes integrados verticalmente (una opción llamada Independent Transmission Operator - ITO). Queda por ver si esta forma de separación funcional será adoptada en algunos países como una alternativa a la separación completa o al modelo ISO y si será efectiva para tratar los problemas de cierre de mercado y discriminación que motivaron la revisión de las Directivas, así como para ayudar a reducir la actual fragmentación regulatoria en Europa.

\subsection{Investigaciones de defensa de la competencia}

La Comisión Europea inició una serie de procedimientos de defensa de la competencia contra empresas del sector energético durante los años 2007, 2008 y 2009, siguiendo los resultados de su Encuesta sectorial. Estos casos se resumen en la Tabla 2 del Anexo. La mayoría de estos casos (todos excepto uno) conciernen a presuntos abusos de posición de dominio, bajo el artículo 82 del Tratado CE (ahora artículo 102 del TFUE).

De los casos de defensa de la competencia presentados en la Tabla 2 se puede observar que todos excepto el caso SvK se refieren a incumbentes integrados verticalmente que son propietarios de la infraestructura de red, a la vez que están presentes en segmentos competitivos de la cadena de suministro (producción y/o suministro minorista); y que la mayoría de los casos se refieren a abusos de exclusión, a través de una variedad de estrategias potenciales de cierre de mercado, incluyendo acceso discriminatorio, contratos a largo plazo, acaparamiento y falta de inversión en las infraestructuras de red. Hasta ahora, la evidencia muestra que la Comisión esta dispuesta a aceptar la resolución de casos (sin aplicar sanciones) a cambio de compromisos significativos. La Comisión ha aceptado medidas tanto de carácter estructural (por ejemplo en los casos E.On, RWE y ENI), como de comportamiento (en los casos Electrabel y GDF, y también en el test de mercado para el caso EDF). Mientras que las medidas estructurales tienen efectos claros en la competencia, no está tan claro que las medidas de carácter no estructural sean igual de eficaces en evitar futuros abusos de posiciones de dominio.

\subsection{El control de fusiones}

La Comisión Europea ha evaluado recientemente varias fusiones significativas en diferentes mercados energéticos nacionales (Portugal, Hungría, Dinamarca, Bélgica, Reino Unido, y Alemania), que se resumen en la Tabla 3 del Anexo. Todas las fusiones recientes en el sector energético excepto una (la adquisición por parte de EDP y ENI de GDP) fueron aprobadas, aunque sujetas en algunos casos a medidas correctivas. Las condiciones impuestas por la Comisión en estas fusiones están en 
línea con las iniciativas europeas actuales para la reforma regulatoria. Por ejemplo, la separación de propiedad formó parte del paquete de medidas de las tres fusiones aprobadas en 2005 y 2006 (y también formó parte de los compromisos propuestos en el caso de EDP/ENI/GDP); y los paquetes de condiciones aceptados por la Comisión Europea también incluyeron desinversiones significativas tanto en casos que involucraban empresas dominantes en sus mercados domésticos (como Suez en Bélgica, y E.On y RWE en Alemania), como en casos que afectaban a empresas sin una posición de claro dominio (como por ejemplo las adquisiciones de EDF en el Reino Unido y en Bélgica).

En principio, el control de fusiones es una herramienta limitada para mejorar los resultados competitivos en los mercados energéticos (puesto que las medidas correctivas pueden ser usadas por las autoridades de defensa de la competencia solo para recuperar las condiciones competitivas existentes antes de la fusión). Por otra parte, en la práctica, el control de fusiones proporciona a la Comisión Europea poder de negociación que puede permitirle impulsar medidas estructurales que de otro modo serían difíciles de lograr.

Una cuestión que sigue en la mesa de debate en relación a fusiones y reestructuraciones en el mercado energético europeo es el papel de las empresas estatales (o empresas con protección estatal). La ley de competencia europea no tiene en cuenta la forma de propiedad de la empresa. Sin embargo, el terreno de juego en términos de control corporativo en el mercado europeo puede estar distorsionado por la presencia de empresas de propiedad estatal. Esto se debe a que las empresas estatales no pueden ser adquiridas, a que pueden tener objetivos distintos al de la maximización de beneficio, y además presentar un conflicto de interés en los sectores regulados (puesto que el sector público se encuentra en ambos lados de la relación regulador-regulado).

\section{Conclusiones: retos futuros para la política energética en la UE}

Este artículo ha examinado las tendencias recientes de los tres pilares fundamentales de la política energética europea (la dependencia energética, la política medioambiental y la competencia). A modo de conclusión, consideramos brevemente los principales desafíos que representan estos tres pilares para el futuro, y qué puede hacer la política energética europea para resolverlos.

Por lo que se refiere a la dependencia energética y a la seguridad del suministro:

- En el mercado europeo existe una clara necesidad de diversificar el mix energético donde sea posible y eficiente, tanto en términos de diferentes tipos de energía como de diferentes fuentes geográficas. Una mayor dependencia de los recursos energéticos domésticos (v.b. las energías renovables y la nuclear) contribuirá a reducir tanto la dependencia del exterior, como a promover los objetivos relacionados con el cambio climático. Esto refuerza la idea de que 
Europa apoye hasta cierto punto las fuentes de energía doméstica, dadas las características de "bien público" que tienen tanto la seguridad energética como el cambio climático. Sin embargo, en el medio plazo, el mercado energético seguirá dependiendo del gas como fuente clave de energía flexible y relativamente limpia, para permitir una transición eficiente hacia un entorno de bajas emisiones de $\mathrm{CO}_{2}$ (donde la nueva energía nuclear, el carbón limpio, y la generación de energías renovables jugarán un papel más importante). Inevitablemente, esto implica que la seguridad del suministro y la dependencia energética seguirán siendo una cuestión clave en el diseño óptimo de la política energética de la UE en un futuro cercano.

- En cuanto a las importaciones de energía, conviene que Europa diversifique su mix de proveedores y reduzca su dependencia de determinados países (especialmente de Rusia). Los nuevos gasoductos y las importaciones de GNL tendrán que desempeñar un papel importante en este sentido. Sin embargo, esto puede ocurrir a costa de tener que adquirir gas de fuentes más caras en condiciones de demanda pico, donde la competencia por los volúmenes de GNL es global.

- Una mayor dependencia de las fuentes de generación intermitentes (como las renovables) crearán nuevos desafíos en términos de seguridad doméstica de suministro, tanto en la electricidad como en el gas. Los periodos de insuficiente generación de energía renovable crearán fuertes picos de demanda de generación térmica y de suministro de gas. Hacer frente a dichos picos requerirá una mayor inversión en infraestructura de red, incluyendo principalmente instalaciones de almacenamiento de gas e infraestructura para su importación. También será necesario un grado suficiente de flexibilidad en los contratos de importación de gas para garantizar la seguridad doméstica de suministro, aunque una vez más, a expensas de un mayor coste para el sistema energético en su conjunto.

Por lo que se refiere al cambio climático y a las políticas relacionadas, los datos y proyecciones que hemos analizado en el presente artículo ilustran el hecho de que el mercado energético en los próximos 10-20 años tendrá que afrontar una transformación profunda para cumplir con los objetivos requeridos. Dados los objetivos ambientales propuestos hay que plantearse de qué forma pueden cumplirse de manera óptima, con el fin de disminuir los costes derivados de la reducción del $\mathrm{CO}_{2}$ (y, eventualmente, de la descarbonización del sector).

La discusión sobre cuál es la mejor manera de alcanzar los objetivos medioambientales plantea una serie de preguntas y retos:

- ¿Qué tecnologías limpias deberían promoverse para minimizar los costes globales de la reducción de emisiones de $\mathrm{CO}_{2}$, y en qué plazo (dada la necesidad de reducir las emisiones de forma progresiva durante un período largo de tiempo)? 
- ¿Cuál es la mejor forma de fomentar las tecnologías bajas en emisiones, sin que los propietarios de esas tecnologías y quién las desarrolla obtengan rentas excesivas, a la vez que proporciona incentivos suficientes a invertir en recursos alternativos? Esto requerirá una comprensión clara de los fallos de mercado relacionados con los factores medioambientales y la promoción de fuentes de energías renovables y otras energías bajas en emisiones, y de la medida en que un sistema de mercado de carbono cap-and-trade como el ETS no es en sí mismo suficiente para abordar estos fallos de mercado.

- ¿Cuál será la mejor manera de utilizar los importantes recursos recaudados mediante la subasta de derechos de emisión de $\mathrm{CO}_{2}$ en el ETS a partir de 2013, de forma que se promueva los objetivos de la política del cambio climático y también se proteja a los consumidores de costes excesivos relacionados con el cambio climático?

En general, está claro que para cumplir con los objetivos medioambientales, el coste global de la energía podría tener que aumentar considerablemente, dada la necesidad de utilizar fuentes de energía más caras (pero más limpias) y de mayor inversión en infraestructura de redes y generación (en particular para hacer frente a la mayor participación de fuentes intermitentes y menos flexibles en el mix energético total). Los precios de $\mathrm{CO}_{2}$ probablemente también aumentarán (para reducir las emisiones de las fuentes de generación de energía con combustibles fósiles), lo que a su vez incrementará el coste de generación (especialmente en las horas de demanda alta) $)^{12}$.

Aunque en el pasado hubo un conflicto entre los objetivos medioambientales y la seguridad de suministro (ya que, con el fin de reducir las emisiones totales, el sector energético utilizó más el gas y menos otras fuentes de energía más fácilmente disponibles a nivel global, como el carbón), es probable que este conflicto se debilite con el tiempo, y que posiblemente desaparezca. Esto se debe al hecho de que para cumplir con los objetivos marcados para combatir el cambio climático, el sector energético europeo tendrá que basarse en mayor medida en las fuentes de energía domésticas (renovables y nucleares), y en menor medida en las de gas. Con el tiempo, el desarrollo comercial de nuevas tecnologías de generación con carbón (v.b., CCS - Carbon Capture and Storage) también contribuirá a alcanzar el doble objetivo de reducir la dependencia energética y producir energía más limpia. Sin embargo, en períodos de demanda alta y baja producción de las fuentes renovables, Europa tendrá que recurrir a la generación con gas (y a las importaciones de gas) como una fuente flexible fundamental de energía. El desarrollo de capacidad do-

${ }^{12}$ El WEO-2009 estima que los precios de $\mathrm{CO}_{2}$ necesitan alcanzar un nivel de $50 \$$ por tonelada en 2020 (y de $110 \$$ en 2030), muy por encima de los precios actuales en Europa que rondaban por debajo de los $20 \$$ en 2009. En su documento de octubre de 2009 (citado anteriormente) Ofgem proyecta que en los escenarios que prevé para el futuro las facturas de energía doméstica se incrementarían en el rango $14-25 \%$ (en términos reales) en el horizonte 2020. 
méstica adicional de almacenamiento de gas será por tanto especialmente importante para lograr los objetivos medioambientales, mientras se gestiona el desafío de la dependencia energética exterior.

Por último, en términos de políticas de competencia, regulación e integración, hay que destacar las ventajas de la integración del mercado energético europeo:

- El poder de mercado de las grandes empresas se reduce al aumentar el tamaño del mercado.

- La amenaza de entrada de otras empresas disciplina a los operadores establecidos.

- El arbitraje estabiliza los mercados y reduce el margen de reserva necesario (fiabilidad operativa) si hay suficiente capacidad de interconexión.

- Empresas más grandes a nivel europeo (pero no necesariamente doméstico) pueden asegurarse el suministro a un coste menor en los mercados internacionales, especialmente si la UE tiene una política energética coordinada internacionalmente.

- Una mayor integración favorece un terreno de juego equilibrado para las empresas que permite la competencia por mérito.

En los últimos años la Comisión Europea ha tenido cierto éxito en las políticas a favor de la competencia. Este ha sido particularmente el caso en las instancias en que tiene competencia exclusiva (casos enmarcados en el artículo 102 del Tratado de Funcionamiento de la UE y fusiones con características transfronterizas). En materia de regulación el progreso ha sido más lento (como refleja la posición de compromiso adoptada en las Directivas 2009), lo que ha llevado a la Comisión a acudir a la política de defensa de la competencia como complemento efectivo (y a veces sustituto) de la regulación. Está por ver si estas Directivas serán efectivas o si se deberá avanzar más decididamente en la segregación de las redes de transporte. Un tema abierto es también el papel de las empresas públicas en los procesos de reestructuración empresarial y fusiones, y su impacto en la integración del mercado.

Mirando hacia un futuro entorno con mayores costes energéticos debidos a los objetivos para combatir el cambio climático y a la mayor necesidad de inversión en infraestructura energética, una aplicación de la política de defensa de la competencia y una mayor integración del mercado europeo seguirán siendo fundamentales para mantener y mejorar la eficiencia del mercado energético, y para garantizar que los precios reflejen de forma fiable las señales económicas y las externalidades medioambientales.

No tiene por que existir un conflicto entre políticas rigurosas de competencia y de medioambiente, a pesar de que las primeras intentan reducir los precios de la energía (hacia los costes), y las segundas es probable que los suban. En particular, será cada vez más importante no confundir los casos de precios de energía altos (v.b., en periodos de demanda pico y baja producción de energía renovable) con el ejercicio de poder de mercado por parte de las empresas. Los precios de la energía deberán 
ser capaces de responder a los picos de demanda para poder proporcionar las señales correctas de escasez económica, promover la eficiencia energética, y premiar la inversión en infraestructura que puede ser sólo o principalmente requerida en condiciones de demanda alta. La aplicación de políticas de defensa de la competencia y/o regulación deberá tener cuidado en no distorsionar estos mecanismos de mercado. En este sentido será importante contar con estructuras de mercado competitivas (en términos de estructura vertical y horizontal).

En relación a la regulación hay que plantear la necesidad de una mayor coordinación de los reguladores nacionales a nivel europeo bajo las mismas directrices que gestione la capacidad de interconexión, trading hubs para gas y la inversión en terminales de GNL, así como el diseño de la red de transporte y los estándares técnicos.

En resumen, consideramos que se debe promover una política energética común, dadas las externalidades entre los países de la UE (mix energético y medio ambiente), las inversiones necesarias en nuevas tecnologías, la necesidad de tener capacidad de negociación frente a los países suministradores, y la conveniencia de contar con una única voz internacional de la UE en esta materia. 


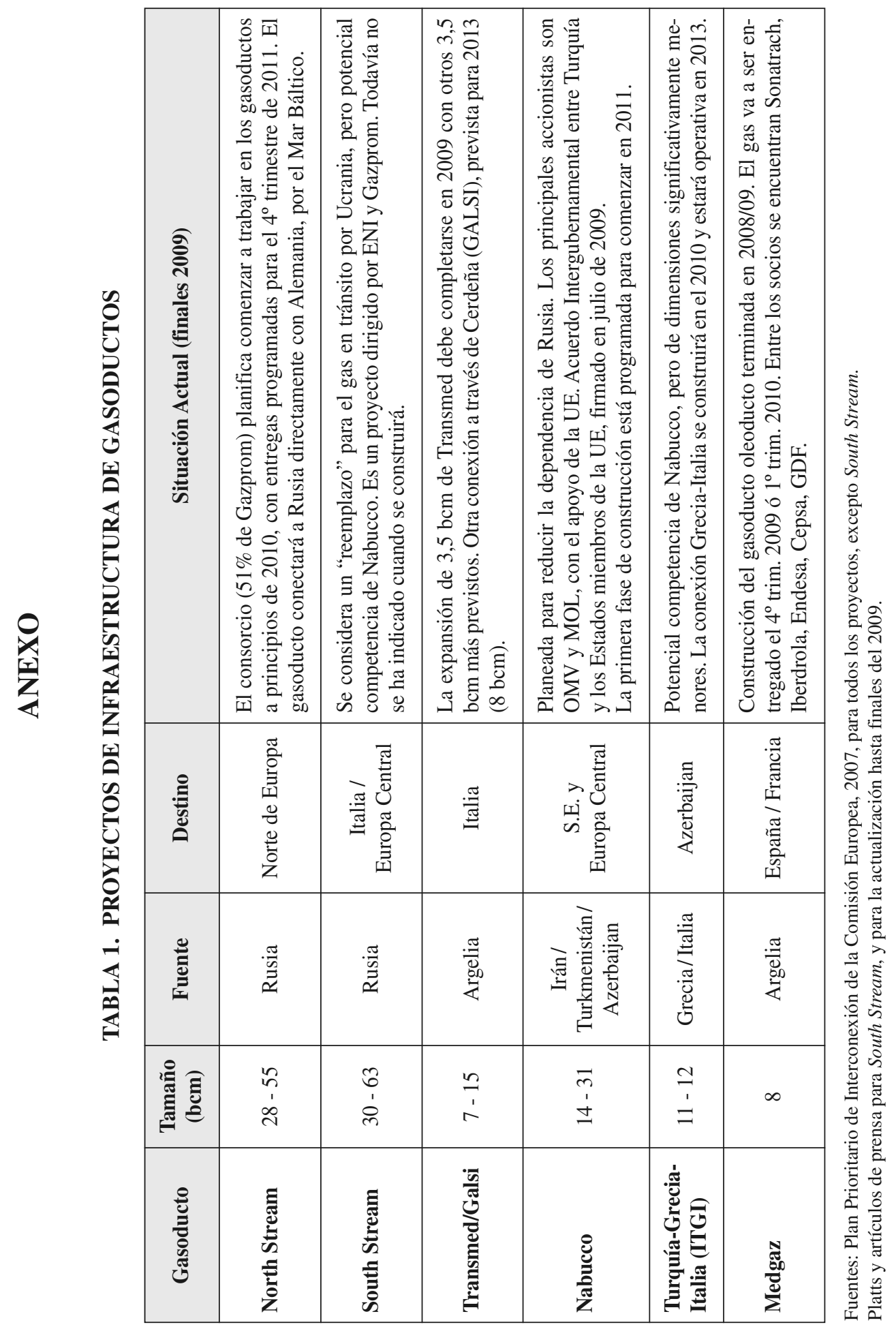




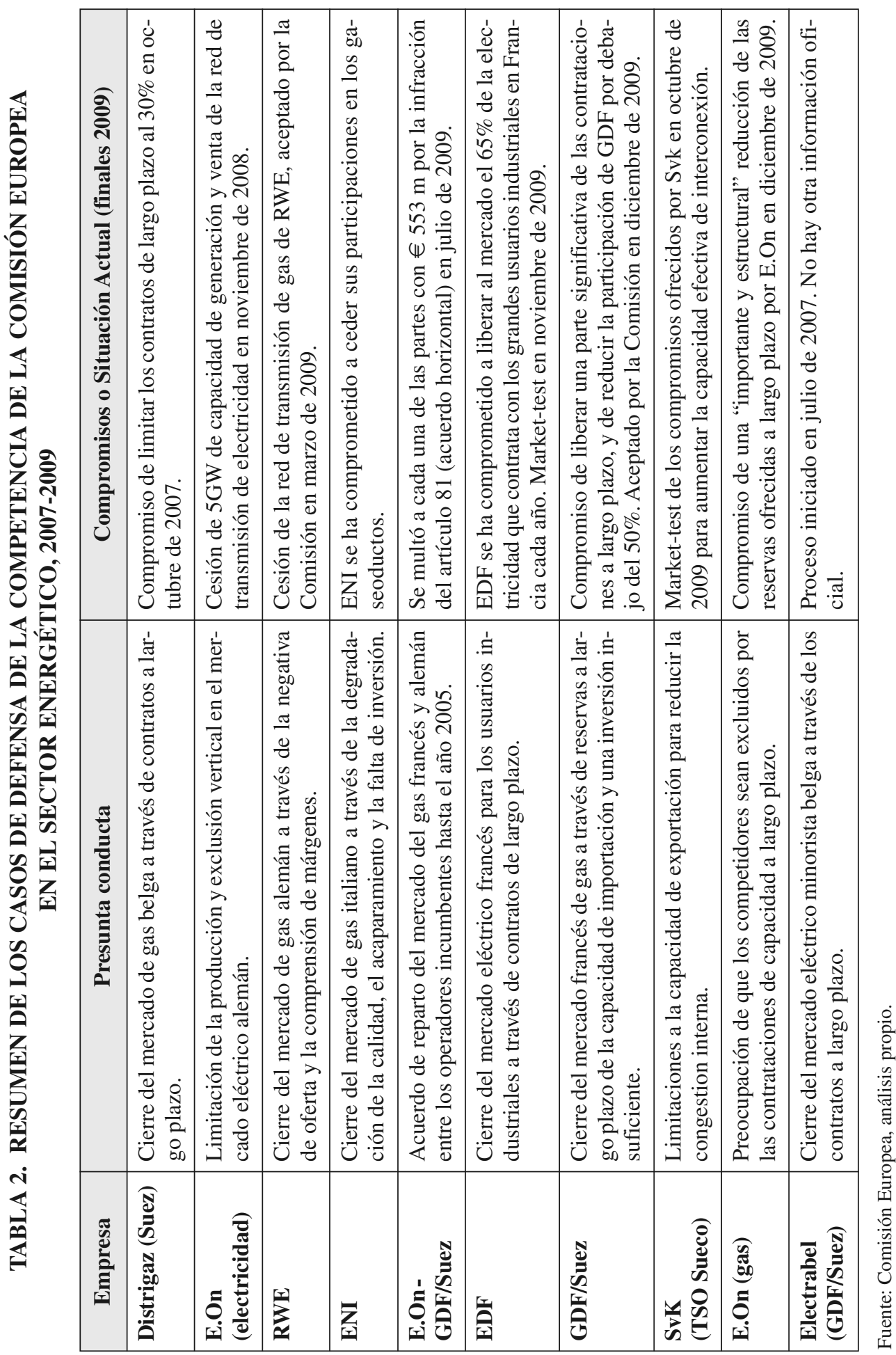




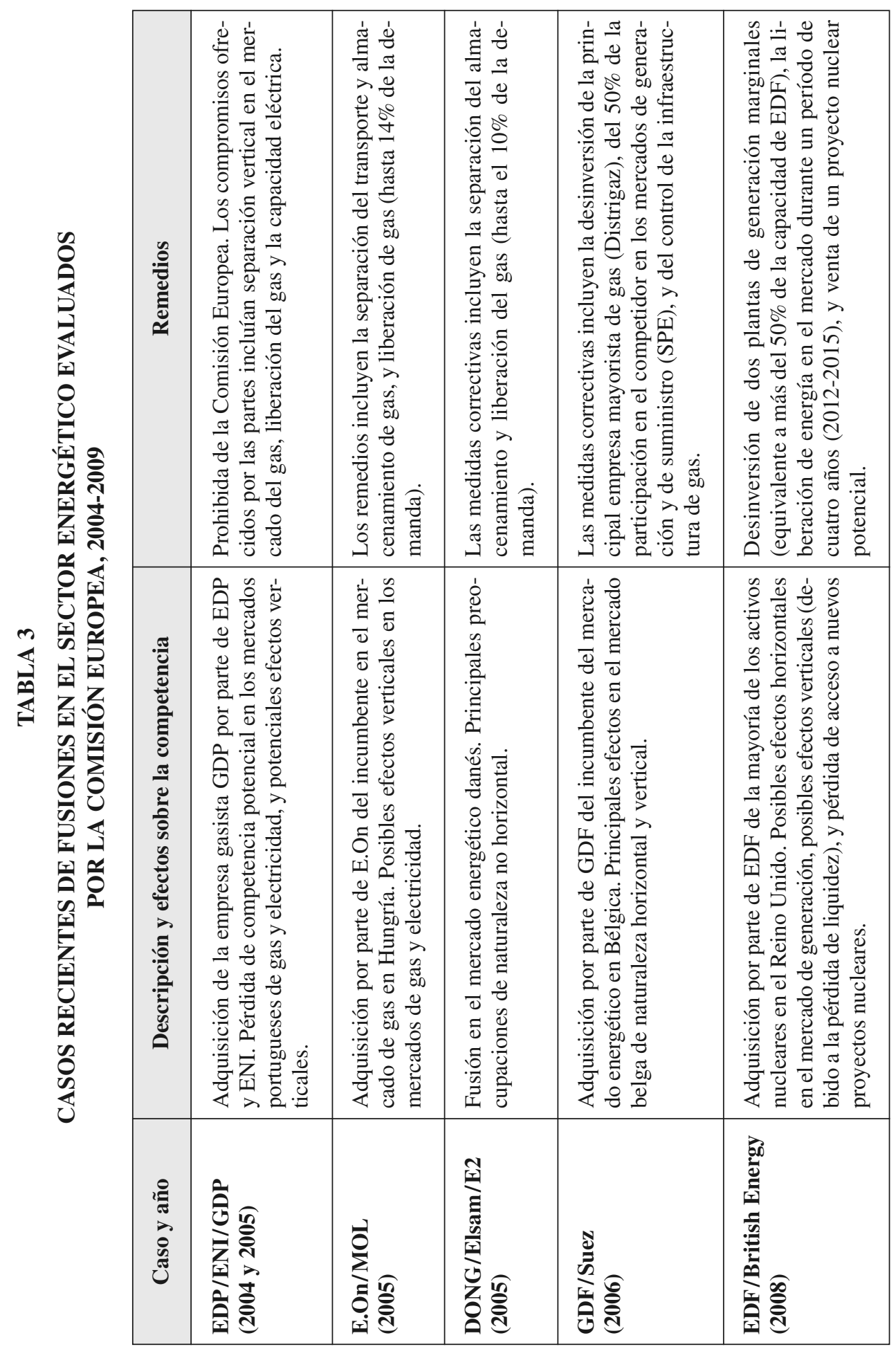




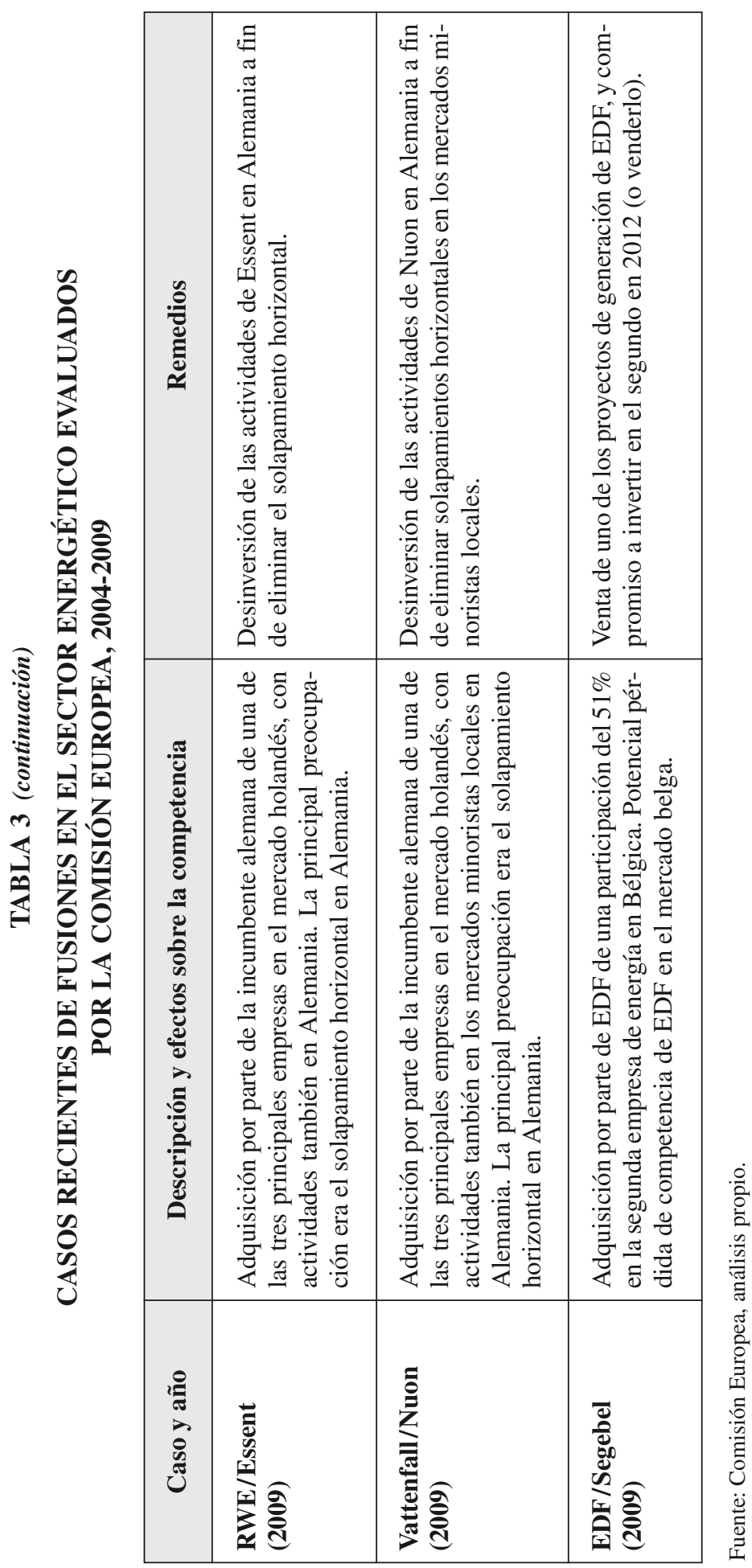


